\title{
ON SOME OPEN QUESTIONS CONCERNING STRICTLY SINGULAR OPERATORS ${ }^{1,2}$
}

\author{
SEYMOUR GOLDBERG AND EDWARD O. THORP
}

T. Kato introduces the following concept of a strictly singular operator in [3].

Definition. Let $X$ and $Y$ be Banach spaces and let $T$ be a bounded linear operator mapping $X$ into $Y . T$ is said to be strictly singular if given any infinite dimensional subspace $M$ of $X, T$ restricted to $M$ is not an isomorphism (i.e., linear homeomorphism).

In particular, every compact operator is strictly singular. Kato proceeds to show that the space $S$ of strictly singular operators possesses some of the important features of the space of compact operators, e.g., $\delta$ is a closed subspace of the space of bounded linear operators from $X$ to $Y$. If $X=Y$, then $\delta$ is a closed ideal. If $X$ and $Y$ are Hilbert spaces, then every strictly singular operator from $X$ to $Y$ is compact.

The following two questions, posed by Kato, are answered below.

(1) Is every strictly singular operator compact?

(2) Is the conjugate of a strictly singular operator strictly singular?

THEOREM. $^{3}$ (a) Every bounded linear operator from $l_{2}$ to $l_{p}$ or from $l_{p}$ to $l_{2}, 1 \leqq p \neq 2, \infty$ is strictly singular.

(b) Let $X$ be a Banach space which does not contain an infinite dimensional reflexive subspace. Then every bounded linear operator mapping $X$ into a reflexive space is strictly singular. Also any bounded linear operator mapping a reflexive space into $X$ is strictly singular.

Remarks. (i) R. S. Phillips exhibited (unpublished) a strictly singular operator which is not compact. Theorem 1 shows that $\delta$ can indeed be a "much larger" space than the space of compact linear operators.

\footnotetext{
Received by the editors March 2, 1962.

${ }^{1}$ Supported, in part, by the National Science Foundation under grant NSF G18052.

${ }^{2}$ Presented at the International Congress of Mathematicians in Stockholm on August 25, 1962.

${ }^{3}$ It has been pointed out to the authors by R. J. Whitley that essentially the same proof used in (a) can be applied to prove that every bounded linear operator from $l_{p}$ to $l_{q}, 1<p, q<\infty, p \neq q$, is strictly singular. As above, $\bar{M}$ is isomorphic to a subspace of $l_{q}$, hence by [1, Theorem 1, p. 194], $l_{q}$ is isomorphic to a subspace of $\bar{M}$ and hence to a subspace of $l_{p}$. This, however, contradicts $[1$, Theorem 7, p. 205].
} 
(ii) $l_{1}$ and $c_{0}$ are spaces which do not contain any infinite dimensional reflexive subspaces. To see this, suppose $M$ were such a subspace of $l_{1}$. Since $l_{1}$ is not reflexive, it is not isomorphic to a subspace of $M$. But by [1, Theorem 1, p. 194], this can only be if $M$ is finite dimensional, which is a contradiction. The argument for $c_{0}$ is the same.

Proof of PART (a). Suppose $1<p \neq 2, \infty$. Let $T: l_{2} \rightarrow l_{p}$ be bounded and linear. Suppose there exists an infinite dimensional subspace of $M$ of $l_{2}$ such that $T$ restricted to $M$ has a bounded inverse. Then $T$ restricted to $\bar{M}$, the closure of $M$ in $l_{2}$, has a bounded inverse so that $\bar{M}$ is isomorphic to a subspace of $l_{p}$. Since $\bar{M}$ is a separable Hilbert space, it is equivalent to $l_{2}$. Thus $l_{2}$ is isomorphic to a subspace of $l_{p}$. This, however, contradicts a theorem due to Banach $[1$, Theorem 7 , p. 205]. The proof that any bounded linear map from $l_{p}$ to $l_{2}, 1<p \neq 2$, $\infty$ is strictly singular is similar to the one just given. Part (b) includes the case for $p=1$.

Proof of Part (b). Let $X$ be as in (b). Suppose $Y$ is reflexive. If $T: X \rightarrow Y$ is bounded and linear but not strictly singular, then $X$ would contain a closed infinite dimensional subspace $N$ which is isomorphic to a closed subspace of $Y$. Thus $N$ is reflexive which cannot be. The same argument can be used to show that $T: Y \rightarrow X$ is strictly singular.

For question (2) we consider the

EXAMPLE. Let $T$ be a continuous linear operator mapping $l_{1}$ onto $l_{2}$. That such a map exists is a result of a theorem of Banach and Mazur $[2$, p. 111], which states that given any separable Banach space $X$, there exists a continuous linear operator which maps $l_{1}$ onto $X$. Now $T$ is strictly singular by the above theorem. However, by $[1$, Theorem 4 , p. 148], $T^{\prime}$ has a bounded inverse. Thus $T^{\prime}$ is not strictly singular.

REMARK. Any bounded linear operator between Banach spaces whose range does not contain any infinite dimensional closed subspace is clearly strictly singular (such operators were considered by R. S. Phillips in his unpublished note). The above example shows that a strictly singular operator need not have this property. The Kato and Phillips concepts coincide when the domain space $X$ is $l_{2}$. Thus whenever the bounded operators from $l_{2} \rightarrow Y$ are strictly singular, they have the Phillips property as well.

The following example shows that a strictly singular operator can have a nonseparable range in contrast to the well-known fact that a compact operator always has a separable range.

EXAMPLE. If $Q$ is an arbitrary set, $l_{p}(Q), 1<p<\infty$, is the space of scalar valued functions $x$ with domain $Q$, having at most countably 
many nonzero coordinates, and such that $\|x\|=\left(\sum_{q \in Q}|x(q)|^{p}\right)^{1 / p}$ is finite. It is a Banach space with this norm. We assert that all the continuous operators $T: l_{2}(Q) \rightarrow l_{p}(Q), 2<p<\infty$, where $Q$ is an uncountable set, are strictly singular and that the inclusion map is such an operator and has a nonseparable range.

(1) To see that all the operators are strictly singular, suppose some $T$ is not. Then there are closed infinite dimensional subspaces $M \subset l_{2}(Q)$ and $N \subset l_{p}(Q)$ such that $T$ restricted to $M$ is an isomorphism between $M$ and $N$. Choose a countable linearly independent subset $\left\{x_{n}\right\}$ of $M$. Then $T$ is an isomorphism between $\operatorname{sp}\left\{x_{n}\right\}$, the set spanned by $\left\{x_{n}\right\}$, and $s p\left\{T x_{n}\right\}$. Let $C$ be $\left\{q \in Q: x_{n}(q) \neq 0\right.$, some $\left.n\right\}$. The set $C$ is countable therefore $R=\left\{x \in l_{2}(Q): x(q)=0, q \notin C\right\}$ is isometrically isomorphic with $l_{2}$. Let $D=\{q \in Q:(T x)(q) \neq 0$, some $x \in R\}$. The set is countable so the set $S=\left\{y \in l_{p}(Q): y(q)=0, q \notin D\right\}$ is isometric to $l_{p}(Q)$. Since $R(T) \subset S$, the restriction of $T$ to $R, T_{R}$, can be regarded as a continuous operator from $l_{2}$ to $l_{p}$. When restricted to $[s p]-\left\{x_{n}\right\}, T_{R}$ is an isometry between $[s p]-\left\{x_{n}\right\}$ and $[s p]-\left\{T x_{n}\right\}$, and they are closed infinite dimensional subspaces of $R$ and $S$ respectively, thus $T_{R}$ is not strictly singular. But this contradicts the first part of the above theorem.

(2) Jensen's inequality shows, just as in the case of $T: l_{2} \rightarrow l_{p}$ in the above theorem, that the inclusion map $I$ is continuous. To see that the range of $I$ is not separable, note that the elements of $l_{p}(Q)$ which are characteristic functions of single points are in the range of $I$. They are mutually separated by distance $2^{1 / p}$ and are uncountable.

REMARK. Arguments similar to that used in (1) show that both parts of the (a) part of the above theorem remain valid when $l_{2}$ and $l_{p}$ are replaced by $l_{2}\left(Q_{1}\right)$ and $l_{p}\left(Q_{2}\right)$ respectively. From the fact that $l_{1}$ and $c_{0}$ contain no infinite dimensional reflexive subspaces, it follows from similar arguments that $l_{1}(Q)$ and $c_{0}(Q)$ do not contain any infinite dimensional reflexive subspaces.

\section{REFERENCES}

1. S. Banach, Operations linéaires, Chelsea, New York, 1955.

2. S. Banach and S. Mazur, Zur Theorie der linearen Dimension, Studia Math. 4 (1933), 100-112.

3. T. Kato, Perturbation theory for nullity, deficiency and other quantities of linear operators, J. Analyse Math. 6 (1958), 261-321.

New Mexico State Universitv 